\title{
AVALIAÇÃO DOS REGIST ROS DE ENFERMAGEM QUANTO AO EXAME FÍSICO
}

\author{
Sandra Patricia da COST A ${ }^{a}$, A driana A parecida PA Z ${ }^{b}$, E miliane N ogueira de SO U ZAc
}

\section{RESUMO}

Os registros de enfermagem geram subsídios essenciais ao planejamento do cuidado individual izado, sendo a coleta de dados a primeira etapa do Processo de Enfermagem. 0 objetivo deste estudo foi avaliar os registros realizados pelos enfer meiros quanto ao exame físico do cliente na unidade de tratamento intensivo (UTI) e na unidade de internação clínica (UIC). 0 estudo é transversal-retrospectivo, no qual for am avaliados 69 prontuários de clientes inter nados em UTI e transferidos para UIC de um hospital público e outro privado. Constatou-se que os registros realizados pelos enfer meiros, mais freqüentes e com melhor qualidade acerca do exame físico, foram os da UTI, enquanto na UIC eram mais frequentes os registros de intercorrências clínicas ocorridas durante o plantão. Evidenciou-se um déficit nos registros acerca do exame físico, o que pode dificultar a assistência individualizada com enfoque nas reais necessidades do cliente, uma vez que muitas alterações podem ser deixadas de serem registradas.

Descritores: Controle de formulários e registros. Exame físico. Registros de enfermagem.

\section{RESUMEN}

L os registros de enfermería son esenciales para la planificación de la atención individualizada, y la recogida de datos el primer paso del proceso de enfer mería. E I objetivo de este estudio fue evaluar los regi stros real izados por los enfer meros refer entes al examen físico del paciente de la U nidad de T ratami entos I ntensivos (U T I) y en la U nidad de Inter nación Clínica (U IC). E I estudio es transversal retrospectivo, donde fueron evaluados prontuarios de pacientes internados en UTI y UIC de un hospital público y otro particular. De los 69 prontuarios evaluados, se verificó que los registr os más frecuentes y con mejor calidad a respecto del examen físi co fuer on los de la U TI, mi entras en la UIC ocur rían más evaluaciones solamente de intercur rencias sufridas por los pacientes. Se evidenció una caída en los registros a respecto del examen físico, lo que puededificultar la asistencia individual y vuelta para las reales necesi dades del paciente, visto que muchas al teraciones dejan de ser apuntadas.

D escriptores: Control de formularios y registros. Examen físico. R egistros de enfermería.

Título: E valuación de los registros de enfermería referentes al examen físico.

\section{ABST RACT}

T he nursing records are essential to generate benefits to individualized care planning, once data collection is the first step of the $\mathrm{N}$ ursing $P$ rocess. The pur pose of this study is to analyze the records made by nurses during each physical patient examination in critical care units (CCU) and hospitalization units ( $\mathrm{HU}$ ). T his is a transverse and retrospective study, in which forms and records from both public and private hospitals w ere anal yzed. F rom the 69 records considered, we observed a greater quality and frequency in records from CCU s, while records from H U s w ere mostly about intercur rences that happened during the shift. The research shows a deficit in physical examination records of patients, which complicates theindividual assistance focused on the real needs of the patient, since many changes in patient's condition might not be recorded.

D escriptors: F orms and records control. P hysical examination. $N$ ursing records. T itle: E valuation of nursing records on the physical examination.

\footnotetext{
${ }^{a}$ Enfermeira assistencial do Hospital da U nimed Nordeste, Caxias do Sul, Rio Grande do Sul, Brasil.

b M estre em Enfermagem, D outoranda pelo Programa de Pós-G raduação em Enfermagem da U niversidade Federal do Rio G rande do Sul (UFRGS), Docente do Curso de Bacharelado em Enfermagem da U niversidade Federal de Ciências da Saúde (U F CSPA) e da Faculdade Nossa Senhora de Fátima, Caxias do Sul, Rio Grande do Sul, Brasil.

'M estre em Ciências da Saúde: Cardiologia, D ocente do Curso de Bacharelado em E nfermagem da U F CSPA e da F aculdade F átima, Caxias do Sul, RS, Brasil.
} 


\section{INT RODUÇÃO}

Os registros em prontuário estabelecem a comunicação escrita de informações pertinentes às condições de saúde-doença do cliente e dos cuidados que são necessários ao mesmo, com a finalidade de assegurar a continuidade da assistência. Contribui, ainda, para a detecção de novos problemas, para a avaliação dos cuidados prescritos e, por fim, possibilita a comparação das respostas do cliente aos cuidados prestados ${ }^{(1)}$.

Deste modo, 0 uso de registros pelos enfermeiros possibilita a mensuração da qualidade da assistência prestada, assim como obtém subsídios para propor mudanças que sejam centradas no cuidado individualizado, integral e resolutivo. $\mathrm{N}$ essa per spectiva, reforça-se que os registros realizados pelos enfer meiros éo instrumento que comprova a qualidade do cuidado, mediante 0 fato de que $50 \%$ das informações inerentes ao cuidado são forneci-das pela enfer magem, tornando-se, assim, indiscutível a importância dos registros sobre 0 processo saúde-doença do cliente em hospitalização ${ }^{(2,3)}$.

N esse contexto, observa-se que os enfer meiros procuram criar e ampliar os espaços de discussões sobre 0 aprimoramento técnico-científico nos diversos campos de atuação, no uso de tecnologias e procedimentos, bem como na maneira do fazer, ser e pensar em Enfermagem. A Resolução do Conselho Federal de Enfermagem (COFEn) no 358/ 2009 preconiza que a Sistematização da Assistência de E nfermagem organiza o trabal ho profissional quanto ao método, pessoal e instrumentos, tornando possível a oper acional ização do P rocesso de Enfermagem (PE $)^{(4)}$. T al processo éum instrumento metodológico que orienta o cuidado a ser prestado pelo profissional de Enfermagem, assim como possibilita o registro da prática assistencial sob a forma de documentação em prontuário do cliente.

Segundo a mesma resolução do COFE n (358/ 2009), cabe ao enfermeiro a lider ança na execução e avaliação do PE, de modo a al cançar os resultados de enfermagem esperados, privativamente, 0 diagnóstico de enfermagem acerca das respostas da pessoa, família ou coletividade em um dado momento do processo saúde-doença, bem como a prescrição das ações de enfermagem a ser em realizadas, em face dessas respostas. Partindo dessa afirmativa, o enfermeiro realiza o exame físico através das habilidades propedêuticas da inspeção, ausculta, pal pação e percussão. T ais habilidades são utilizadas para realizar a primeira etapa do PE, denominada de coleta de dados, que se subdivide em anamnese ou entrevista (dados subjetivos) e exame físico (dados objetivos). Portanto, essas informações são fundamentais para o desencadeamento das etapas do PE, que compreende: a) histórico de enfermagem (anamnese e exame físico); b) diagnósticos de enfermagem; c) plano ou planejamento de cuidados; d) implementação; e) aval iação(4). 0 exame físico deve ser criterioso, no sentido de identificar os problemas de enfer magem ao utilizar as habilidades propedêuticas, de validar as informações coletadas pela anamnese (entrevista), e em subsidiar as demais etapas do PE.

Face ao exposto, tão importante quanto à realização do exame físico é o registro do enfer meiro sobre essas informações que oportunizam cuidados que são peculiares ao processo saúde-doença, de tal modo que sejam individualizados, resolutivos e atendam o cliente na sua integralidade. N esta perspectiva, em face da lacuna de conhecimento diante dos escassos estudos que abordam a temática, esta investigação teve por objetivo avaliar os registros realizados pelos enfermeiros quanto ao exame físico do cliente na unidade de tratamento intensivo (UTI) e na unidade de inter nação clínica (UIC).

\section{MATERIAIS E MÉTODOS}

Trata-se de um estudo retrospectivo, com delineamento transversal. 0 estudo foi realizado em duas instituições hospitalares, sendo uma filantrópica e outra pública. Tais instituições tem múltiplas especialidades, e situam-se no município de Caxias do Sul, Rio Grande do Sul. A escolha dessas instituições ocorreu pelo fato de que ambas são instituições vinculadas ao ensino e pesquisa que recebem acadêmicos de enfermagem.

A coleta de dados iniciou com busca ativa no sistema de internação da UTI para que a partir dos prontuários de clientes que for am transferidos para a UIC, nos meses de novembro e dezembro de 2007, fosse possível a anál ise compar ativa entre os registros dessas unidades, em dois momentos distintos de atenção (UTI e UIC) de um mesmo cliente, pois as informações relacionadas ao exame físico requer em registro em ambos os cenários de inter nação. A partir de então, foram solicitados os 
prontuários junto ao serviço de arquivo médico das duas instituições.

A amostra foi composta, consecutivamente, por 69 prontuários que apresentaram os seguintes critérios de inclusão: cliente inter nado nos meses de novembro ou dezembro na UTI; internação maior que 24 horas em U TI; ter a transferência da UT I para UIC; adultos $\geq$ de 18 anos; evolução mais completa nas últimas 24 horas de internação hospitalar, ou seja, com descrição de maior número de itens relacionados aos sistemas orgânicos; e prontuários com os registros das evoluções de enfermeiros legíveis, incluindo data e hora, além da assinatura legível ou carimbo. Foram considerados critérios de exclusão: óbito na UTI, al ta hospital ar da UTI sem transferência a U Cl, alta hospitalar nas primeiras $24 \mathrm{~h}$ de internação na UIC.

A aval iação dos registros dos enfer meiros nos prontuários ocorreu em dois momentos distintos: 1) após as primeiras $24 \mathrm{~h}$ de inter nação do cliente na UTI; 2) após as primeiras $24 \mathrm{~h}$ de internação na UIC. Os dados dos prontuários for am coletados por meio de um instrumento próprio, do tipo checklist, com questões sobre a aval iação do exame físico. T al instrumento foi elaborado com base em revisão bibliográfica ${ }^{(5,6)}$, em que foram extraídos os dados de maior relevância, em se tratando de exame físico direcionado aos sistemas: neurológico, pulmonar, cardiovascular, gastrintestinal, geniturinário e pele e mucosas. Para cada item avaliado, as respostas foram categorizadas em: "registrado" ou "não registrado", com base na descrição objetiva relacionada aos parâmetros dos sinais vitais, reações e características apresentadas à aplicação da semiologia e semiotécnica para revisão dos referidos sistemas.

Os sistemas orgânicos afetados for am definidos conforme os diagnósticos médicos principais que motivaram a inter nação dos pacientes nas referidas instituições. 0 instrumento também contemplou dados relativos à descrição da presença de cateteres, drenos e sondas, mediante conferência da prescrição médica da mesma data, para verificar se existia a prescrição, 0 aprazamento de medicamentos injetáveis por via endovenosa (contínua ou intermitente) e os indicativos de presença de cateteres, sondas ou drenos (prescrição médica de medicação, dieta, irrigação, entre outros, ou prescrição de cuidados de enfermagem). Caso não houvesse registro relacionado ao item, era marcada a resposta "não se aplica".
A partir de então, foi submetido à validação de face, sendo realizada através da submissão do instrumento a um grupo de profissionais, sendo consider ados especialistas na temática. Selecionaram-se quatro pesquisadores/ enfer meiros, dos quais dois eram docentes com titulação de mestre e que lecionavam disciplinas de semiologia e semiotécnica em cursos de graduação de enfermagem (teoria e prática) há pelo menos três anos, e dois enfer meiros assistenciais, ambos com cargo de gerência das unidades: um da unidade de intensivismo e o outro de unidade clínica, ambos especialistas e com ampla experiência em assistência direta ao cliente e domínio do PE. Salienta-se que os profissionais não eram colaboradores dos hospitais em que foi realizado este estudo.

A avaliação desse grupo de profissionais teve por objetivo identificar se as questões elaboradas estavam de fato adequadas ao objetivo do estudo, bem como as terminologias e expressões utilizadas na elaboração do instrumento de coleta de dados. A pós a avaliação do grupo, por meio de um formulário solicitando consider ações acerca da organização dos itens, prioridade de registro, clareza dos ter mos e compreensão do conteúdo, for am realizados al guns ajustes no instrumento.

A verificação das técnicas propedêuticas utilizadas pel os enfermeiros para realizar o exame físico ocorreu a partir dos dados objetivos registrados nas evoluções de enfermagem, que eram textos livres, e que foram assinaladas em uma das seguintes técnicas: inspeção, palpação, ausculta ou percussão. Exemplifica-se com alguns registros seguidos pela técnica propedêutica(6), marcada no instrumento de coleta de dados: paciente lúcido e orientado - inspeção; pulsos cheios e simétricos pal pação; ruídos hidroaéreos - ausculta. No entanto, antes da coleta de dados propriamente dita, foi realizado um teste-piloto, com três prontuários de cada instituição, a fim de testar o instrumento para adequação. As pesquisador as anal isaram e discutiram novamente as questões, realizando algumas alterações, para sua mel hor aplicação. Os dados foram coletados nos meses de fevereiro e março de 2008.

Os dados foram analisados com auxílio do programa Statistical Package for Social Sciences (SPSS), versão 12.0. As variáveis categóricas foram expressas em freqüências absolutas (n) e relativas (\%) e, as variáveis contínuas, através de média e desvio padrão ou mediana e percentis 25 e 75, 
em consonância com os pressupostos de normalidade. Para comparação entre as médias, foi utilizado 0 teste de te Student, sendo consider ado significativo um $P<0,05$. E ste estudo foi aprovado pelo Comitê de Ética em Pesquisa das duas instituições. Para tanto, os pesquisadores assinar am um T ermo de Compromisso para coleta e utilização de dados de prontuário. Essa investigação está registrada sob os números 62/ 2007 e 32/ 2008.

\section{RESULT ADOS}

A amostra foi constituída por um total de 69 prontuários, sendo que a maioria era de clientes do sexo feminino (58\%). Os sistemas orgânicos mais afetados foram o cardiovascular e o pulmonar, juntos responsáveis por $76,8 \%$ dos motivos das inter nações em UTI. E stes e outros dados estão descritos na T abela 1.

T abela 1 - Características dos clientes descritas em prontuário ( $n=69)$. Caxias do Sul, RS, 2008.

\begin{tabular}{lc}
\hline Características & $\mathbf{n}(\%)$ \\
\hline Idade* & $58,6 \pm 17,1$ \\
Sexo feminino & $40(58,0)$ \\
Instituição pública & $51(73,0)$ \\
Sistemas orgânicos afetados & \\
$\quad$ Cardiovascular & $40(58,0)$ \\
Pulmonar & $13(18,8)$ \\
N eurológico & $7(10,1)$ \\
E ndócrino/ metabólico & $4(5,8)$ \\
Locomotor & $3(4,5)$ \\
N efrológico/ urológico & $2(2,9)$ \\
\hline
\end{tabular}

* média \pm desvio padrão.

Quanto ao turno que mais apresentou registros do examefísico (EF) pelo enfer meiro em prontuários, na UT I, foi o tur no da tarde com $30(43,5 \%)$ prontuários, seguido pelo turno da noite com 22 $(31,9 \%)$, e o turno da manhã com 17 (24,6\%). Já na UIC, o turno da tarde apresentou 28 (40,6\%) prontuários com registro do $\mathrm{EF}$; o turno da manhã apresentou $14(20,3 \%)$ e, o turno da noite 10 (14,5\%), sendo que $14(20,3 \%)$ prontuários não apresentavam evolução de enfer magem com registro do EF durante a permanência do cliente nessa unidade, e $3(4,3 \%)$ prontuários não apresentavam data e hora do registro do enfermeiro.

$\mathrm{Na}$ UT I todos os prontuários anal isados apresentavam evolução de enfermagem realizada pelo enfermeiro, mas, após a transferência para a U IC, somente 53 (76,8\%) prontuários apresentavam esse registro após as primeiras $24 \mathrm{~h}$ de permanência na referida unidade (10 prontuários da instituição pública e 06 da instituição privada com ausência de registro). 0 sistema orgânico que mais apresentou registro na UT I foi o respiratório, com dados relativos à avaliação do padrão respiratório $(95,6 \%)$, seguido pelo sistema neurológico, com dados do nível de consciência (84\%). Na UIC, os sistemas que apresentaram maior número de registros foi o respiratório, por meio da avaliação do padrão respiratório; pele e mucosas por meio da avaliação da integridade cutâneo-mucosa, ambos com $81,1 \%$ (T abela 2).

T abela 2 - Registro do exame físico em prontuário. Caxias do Sul, RS, 2008.

\begin{tabular}{|c|c|c|}
\hline Sistemas & $\frac{\text { UT I }(n=69)}{n(\%)}$ & $\frac{\text { UIC }(n=53)}{n(\%)}$ \\
\hline \multicolumn{3}{|l|}{ Neurológico } \\
\hline Nível de consciência & $58(84,0)$ & $34(64,2)$ \\
\hline Função motora & $9(13,0)$ & $4(7,5)$ \\
\hline \multicolumn{3}{|l|}{ Pulmonar } \\
\hline Padrão respiratório & $66(95,6)$ & $43(81,1)$ \\
\hline Ausculta pulmonar & $47(68,1)$ & $26(49,1)$ \\
\hline \multicolumn{3}{|l|}{ Cardiovascular } \\
\hline A usculta cardíaca & $34(49,3)$ & $27(50,9)$ \\
\hline Perfusão periférica & $60(86,9)$ & $42(79,2)$ \\
\hline \multicolumn{3}{|l|}{ G eniturinário } \\
\hline Eliminação urinária & $57(82,6)$ & $42(79,2)$ \\
\hline \multicolumn{3}{|l|}{ G astrintestinal } \\
\hline E stado nutricional & $33(47,8)$ & $36(67,9)$ \\
\hline Ruídos hidroaéreos & $58(84,0)$ & $36(67,9)$ \\
\hline T ensão abdominal & $60(86,9)$ & $36(67,9)$ \\
\hline Eliminações intestinais & $31(44,9)$ & $30(56,6)$ \\
\hline \multicolumn{3}{|l|}{ Pele e M ucosas } \\
\hline Hidratação & $54(78,3)$ & $40(75,5)$ \\
\hline $\begin{array}{l}\text { Integridade cutâneo- } \\
\text { mucosa }\end{array}$ & $58(84,0)$ & $43(81,1)$ \\
\hline
\end{tabular}

Legenda: UTI: U nidade de Tratamento Intensivo; UIC: U nidade de Internação Clínica.

E $m$ relação ao registro da presença de acesso venoso, tanto periférico como central, dos 64 $(92,7 \%)$ clientes que tinham prescritas medicações por via endovenosa, durante internação na UTI, somente $50(78,1 \%)$ prontuários apresentaram 0 registro, e na UIC apenas 22 (62,85\%) prontuários dos 35 clientes com a mesma prescrição. Quanto à presença de drenos ou sondas (nasogástrica, nasoenteral ou vesical), 25 (59,5\%) dos 42 pacientes, e 
$18(66,7 \%)$ dos 27 pacientes tinham indicativos de presença de, pelo menos, uma sonda, sendo estes descritos nos registros dos enfermeiros na UTI e UIC, respectivamente. Já na análise dos registros acerca da infusão endovenosa de medicações contínuas, foram encontrados em 50 (86,2\%) prontuários dos 58 clientes inter nados na U TI e $18(64,3 \%)$ prontuários dos 28 clientes da UIC que estavam recebendo medicação contínua por tal via.

De acordo com a descrição do exame físico nos registros dos enfermeiros analisados por unidade, a maioria estava relacionada à técnica propedêutica da inspeção, com uma média de $7,12 \pm 2,3$ itens descritos durante inter nação em UTI e 6,55 2,5 em UIC, seguidos pela pal pação e ausculta. Em relação à percussão, não foi identificado nenhum dado registrado pelo enfermeiro nas evoluções. A diferença entre as médias do número de itens registrados não foi significativamente diferente entre as evoluções da UTI e UIC. Dados demonstrados na T abela 3.

T abela 3 - N úmero de itens registrados em evolução, de acordo com a técnica propedêutica utilizada. Caxias do Sul, RS, 2008.

\begin{tabular}{lcccc}
\hline T écnicas & UT I $(\mathbf{n}=\mathbf{6 9})$ & UIC $(\mathbf{n}=53)$ & \multirow{2}{*}{ P* } \\
\cline { 2 - 4 } & Média $\pm \mathbf{D P}$ & & Média $\pm \mathbf{D P}$ & \\
\hline Inspeção & $7,12 \pm 2,3$ & $6,55 \pm 2,5$ & 0,19 \\
Palpação & $2,52 \pm 0,8$ & $2,25 \pm 1,1$ & 0,12 \\
Ausculta & $2,0 \pm 0,9$ & $1,7 \pm 1,2$ & 0,10 \\
Percussão & - & - & - \\
\hline
\end{tabular}

* T este $t$ de Student.

Legenda: UTI: U nidade de T ratamento Intensivo; UIC: U nidadede Internação Clínica; D P: desvio padrão.

\section{DISCUSSÃO}

Este estudo avaliou a qual idade dos registros dos enfer meiros acerca da realização do exame físico por meio das evoluções de enfermagem, por ser considerado parte de uma etapa essencial do $P E$, na medida em que busca identificar ou confirmar possíveis alterações, tanto de melhora quanto de piora do estado de saúde do cliente ${ }^{(7,8)}$. Quando realizado periodicamente 0 PE, torna-se possível avaliar o progresso do cliente e as suas respostas frente às intervenções instituídas. Portanto, ao incorporar a Sistematização da Assistência de Enfermagem (SAE) na prática de enfermagem através do $P E$, considera-se que, quanto mais o enfer- meiro souber sobre semiologia e semiotécnica clínica, mais subsídios terá para planejar com qualidade e eficácia a assistência a ser prestada ao cliente de maneira individual, resolutiva e integ ral $(9,10)$. A utilização do PE é uma exigência estabelecida pelos padrões da prática nacional ${ }^{(11)}$, e seus princípios e regras são destinados a promover o pensamento crítico e reflexivo no cenário clínico de enfer magem ${ }^{(7,12)}$, pois subsidia a assistência a ser prestada ao cliente e família.

T odavia, a infor mação não registrada é informação perdida, e a escassa documentação sugere má prática na enfermagem ${ }^{(10)}$. E ntretanto, sabe-se que, nos E stados U nidos, os registros, al ém de denotarem a qualidade dos cuidados de enfer magem, subsidiam os financiamentos e reembolsos pelas instituições financiadoras, tor nando-se um dos temas mais desafiadores para os profissionais da enfermagem ${ }^{(8)}$. No Brasil, os registros de enfermagem são considerados um importante instrumento para ser consultado em situações de processos judiciais que envolvem aspectos legais, éticos, científicos e educacionais. Os registros de enfermagem completos consistem em um dos mais importantes indicadores de qualidade do cuidado de enfermagem ${ }^{(2,7)}$. A ssim, constata-se que, apesar da maioria dos clientes ter internado em decorrência de alterações do sistema cardiovascular e pulmonar, os registros acerca desses sistemas corporais ainda precisam ser melhorados.

Outro aspecto importante são os registros referentes ao sistema neurológico, pois em ambas as unidades nem todas as evoluções apresentavam o registro acerca do nível de consciência do cliente, sendo que esse é um dado objetivo que deve ser realizado na avaliação de todos os clientes. $M$ enciona-se ainda que, relacionados à função motora, os registros foram encontrados em pequena parte dos prontuários, enquanto os clientes estavam inter nados nas unidades cenários deste estudo.

Os registros das condições acerca da mobilidade física e do grau de dependência dos clientes tornam-se relevantes, na medida em que auxilia no dimensionamento de pessoal e adequação de recursos. Outro aspecto importante foi em relação às técnicas propedêuticas mais utilizadas pelos enfer meiros, sendo a inspeção ea pal pação as que mais se destacaram por meio do registro nos itens nível de consciência, padrão respiratório, perfusão periférica, tensão abdominal e integridade cutâneomucosa. 
$\mathrm{Na}$ utilização da ausculta para a realização do exame físico, pode-se observar que há uma lacuna nos registros, pois as menores frequências foram observadas na ausculta pulmonar e cardíaca, lembrando que os sistemas cardiovascular erespiratório são os responsáveis pela maioria das inter nações e podem estar diretamente envolvidos na instabilidade do quadro clínico do cliente. No entanto, não foram encontrados, nas evoluções dos enfermeiros, dados obtidos por meio da percussão. $T$ al habilidade propedêutica pode ser influenciada pelo ensino da semiologia e semiotécnica nos cursos de Graduação em Enfermagem. Relacionado a isso, um estudo realizado em São Paulo, com o objetivo de verificar o ensino de exame físico em escolas de G raduação de E nfermagem, constatou que, dentre os fatores dificultadores do ensino desse conteúdo, destacavam-se o conhecimento insuficiente em percussão $(46,1 \%)$, ausculta $(43,6 \%)$ e palpação $(35,9 \%)$, por parte dos docentes $^{(13)}$.

Outros itens com déficit nos registros foram a presença de cateteres, drenos/ sondas e a infusão de drogas. Esses registros tornam-se relevantes devido à ocorrência não rara de eventos adversos, como flebite, infiltr ações, posicionamento inadequado e suas consequentes implicações de ordem clínica e legal. N o entanto, ao analisar os prontuários da UTI, foram constatados registros dos dados mais frequentes e com mel hor qualidade acerca do exame físico quando comparado à UIC. Na UTI todos os prontuários analisados apresentavam registros de enfermeiros, enquanto na UIC eram mais frequentes os registros apenas das intercorrências sofridas pel os clientes. U m dos fatores que pode ter contribuído para esse resultado, é o fato da UTI ser considerada um setor de al ta complexidade, que admite clientes potencialmente graves ou com alteração de um ou mais sistemas orgânicos. A ssim, é possível que os enfermeiros tenham aderido à realização do registro do exame físico como meio facilitador para o desenvolvimento da assistência, em decorrência da instabilidade e da potencialidade de complicações do quadro clínico dos clientes.

Entretanto, torna-se relevante para o enfermeiro prestar atenção aos relatos do cliente e direcionar ao exame físico, de modo que um quadro claro seja el aborado a respeito de sua condição de saúde, independente do setor em que esteja sen- do cuidado. Isto significa que a aplicação do exame físico exige paciência, habilidade técnica e comunicação interpessoal, bem como competência clínica por parte do profissional de enfermagem ${ }^{(5,11,15)}$.

Face ao exposto, os dados deste estudo possibilitam uma reflexão acerca da importância da realização do registro do exame físico em evoluções diárias real izadas pel os enfer meiros durante o cuidado ao cliente, bem como da necessidade de implementação e qualificação dos mesmos, pois constituem a base para as demais etapas do PE. Corroborando, dados de um estudo que objetivou classificar os conteúdos dos registros de enfermagem de acordo com as necessidades humanas básicas, demonstrar am que $68,2 \%$ do conteúdo das anotações estão relacionadas às necessidades psicobiológi$\operatorname{cas}^{(16)}$.

Importante ainda mencionar que, diante da complexidade do ensino do exame físico e da importância do enfermeiro dominar a semiologia e semiotécnica para possibilitar uma coleta de dados abrangente, quanto mais diversificadas forem as maneiras de ensinar sobre um mesmo assunto, mais será favorecida a aprendizagem, pois estará presente a promoção do desenvolvimento de várias habilidades dos futuros enfermeiros ${ }^{(17)}$. U m outro aspecto que merece destaque é o fato de que os registros informatizados, com um conjunto mínimo de dados estruturados, costumam ser preenchidos por completo, conferindo maior qualidade a essa etapa do PE ${ }^{(18,19)}$.

E m consonância, uma revisão sistemática, real izada por pesquisadores britânicos com o objetivo de analisar a relação entre a prática de enfermagem e o registro da prática, evidenciou aspectos prós e contras para diferentes estratégias de registros: tex to livre em papel, infor matizado e por gravação de voz. E m relação ao registro informatizado, além da vantagem mencionada anteriormente, destacou-se a necessidade de maior tempo para sua execução, principalmente no período de implantação do sistema, quando a equipe está em fase de adaptação. 0 texto livre em papel pode ser útil para as práticas de enfermagem que envolvem aspectos psicossociais como, por exemplo, saúde da mulher, da criança e do adolescente e psiquiatria. Quanto à gravação de voz, os enfermeiros vêem essa estratégia como o cumprimento de uma atividade formal, que visa à proteção legal(20). 


\section{CONCLUSÕES}

Evidenciou-se um déficit na qualidade dos registros acerca do exame físico, na medida em que nem todos os prontuários das duas instituições apresentaram o exame físico registrado pelo enfermeiro. T al evidência vem a configurar um desafio para a enfermagem, pois a escassez de dados ou a realização incompleta poderá dificultar a assistência individualizada, voltada às reais necessidades do cliente, uma vez que muitas alter ações deixam de ser identificadas e registradas.

Os resultados deste estudo contribuem para a reflexão acerca dos registros de enfermagem como outros estudos já publicados $s^{(3,9,13,16)}$, que apontam uma lacuna no "fazer" do enfermeiro, principal mente em unidade de inter nação, quando comparada à unidade de cuidados mais complexos, como a UTI. Desta maneira, torna-se necessário repensar as práticas de enfer magem quanto ao registro do exame físico, sendo fundamentando por conhecimentos básicos e essenciais. Assim, novas propostas estratégicas para modificar esta realidade precisam ser pensadas e discutidas, seja por meio de cur sos de extensão, aprimoramento, capacitações em serviço, grupos de discussão sobre os registros de enfermagem e/ ou incentivos institucionais. Espaços de socialização/ discussão que abordem essa temática constituem-se como estratégias para a obtenção da qualidade dos registros de enfermagem, caracterizando-se como um diferencial no pensar efazer da prática profissional do enfer meiro. Contudo, existe a necessidade de estudos prospectivos e comparados para se avaliar possíveis fatores que influenciam a qualidade dos registros e consequentemente, da assistência de enfermagem.

\section{REFERÊNCIAS}

1 Cianciarullo T I, G ualda D M R , M elleiro M M , Anabuki M H . Sistema de assistência de enfermagem: evolução e tendências. São Paulo: Í cone; 2001.

2 Luz A, M artins A P, D ynewicz A M . Características de anotações de enfermagem encontradas em auditoria. Rev E letrônica E nferm [ I nter net] . 2007 [ citado 2007 set 5] ;9(2):344-61. Disponível em: http:/ / www.fen. ufg.br/ revista/ v9/ n2/ v9n2a05.htm.

3 M atsuda L M , Silva D M P, É vora Y D M , Coimbra AH . A notações/ registros de enfermagem: instrumento de comunicação para a qualidade do cuidado? Rev Ele- trônica Enferm [ Internet] . 2006 [ citado 2009 out 16] ;8(3):415-21. Disponível em: http:/ / www.fen.ufg. br/ revista/ revista8_3/v8n3a12.htm.

4 Consel ho F ederal de E nfermagem. Resolução COFE N ำ 358, de 15 de outubro de 2009: dispõe sobre a Sistematização da A ssistência de E nfer magem e a implementação do Processo de E nfer magem em ambientes, públicos ou privados, em que ocorre o cuidado profissional de Enfermagem, e dá outras providências. Brasília (DF); 2009.

5 Barros A L BL , A ndriolo A, I sabella A PJ, Sá AC, Bettencourt ARC, Caseiro AC, et al. A namnese e exame físico. Porto Alegre: Artmed; 2005.

6 Seidel H M , Ball JW, Dains JE, Benedict GW. M osby guia de examefísico. 6å ed. Rio de Janeiro: Elsevier;2007.

7 F reitas M C, Queiroz TA, Souza JAV. 0 processo de enfer magem sob a ótica das enfermeiras de uma maternidade. Rev Bras Enferm. 2007;60(2):207-12.

8 Capernito-M oyet $L J$. Compreensão do processo de enfermagem: mapeamento de conceitos e planejamento do cuidado para estudantes. Porto A legre: A rtmed; 2007.

9 Patine FS, Barboza DB, Pinto M H. Ensino do exame físico em uma escola de enfermagem. A rq Ciênc Saúde [ I nternet] . 2004 [ citado 2007 ago 02] ;11(2). D isponível em: http:/ / www.cienciasdasaude.famerp.br/ racs_ol/Vol-11-2/ ac11\%20-\%20id\%2015.pdf.

100 choa-Vigo K, PaceA E, Rossi LA, H ayashida M . Avaliação da qualidade das anotações de enfermagem embasadas no processo de enfermagem. Rev Esc Enferm USP. 2001;35(4):390-8.

11 H ermida PM , A raújo IE M . E labor ação e validação do instrumento de entrevista de enfermagem. Rev Bras Enferm. 2006;59(3):314-20.

12 Conselho Federal de Enfermagem. Resolução COFEN n² 272, de 27 de agosto de 2002: dispõe sobre a Sistematização da A ssistência de E nfer magem - SAE - nas Instituições de Saúde Brasileiras. Rio de Janeiro; 2002.

13 Alfaro-L efevre R. A plicação do processo de enfermagem: promoção do cuidado colaborativo. Porto Alegre: Artmed; 2005.

14 Sousa VD, Barros ALBL. 0 ensino do exame físico em escolas de graduação em enfer magem do município de São Paul o. Rev L atino-A m E nfermagem. 1998; 6(3):11-22. 
15 Santos SR, Paula AFA, Lima JP. 0 enfermeiro e sua percepção sobre o sistema manual de registro no prontuário. Rev Latino-Am Enfermagem. 2003;11(1):80-7.

16 De Carval ho CJA, N ogueira M S. A nálise dos registros de enfermagem em uma unidade cirúrgica de um hospital-escola público. Rev Gaúcha Enferm. 2006;27(4):584-9.

17 N akatani AY K, Campos E C, Bachion M M . 0 ensino do exame físico pulmonar através do método de problematização. Rev L atino-Am E nfermagem. 2000; 8(6):117-23.
18 D aly J M , Buckwalter K, M aas M. W ritten and computerized care plans: organizational processes and effect on patient outcomes. J Gerontol N urs. 2002; 28(9):14-23.

19 A mmenwerth E, Eichstädter HR, Pohl U, Rebel S, Ziegler S. A randomized evaluation of a computerbased nursing documentation system. M ethods Inf M ed. 2001;40(2):61-8.

20 U rquhart C, Currell R. Reviewing the evidence on nursing record systems. H ealth I nformatics J. 2005; 11(1):33-44.
Endereço da autora / D irección del autor /

\section{Author's address:}

E miliane N ogueira de Souza

Rua J osé Gollo, 192, ap. 803, M al. F loriano

95020-090, Caxias do Sul, RS

E-mail:enogsouza@hotmail.com
Recebido em: 21/ 11/ 2009

A provado em: 09/ 03/ 2010 